\author{
Military Technical College \\ Kobry El-Kobbah, \\ Cairo, Egypt
}

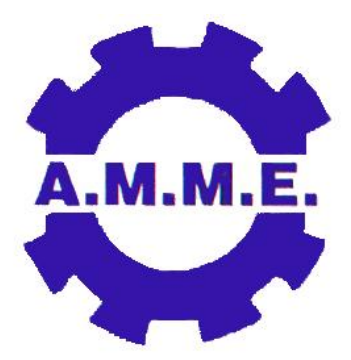

14th International Conference on

Applied Mechanics and

Mechanical Engineering.

\title{
Finite Element Analysis of Fatigue Damage of Composite Laminated Structures under Stochastic Loading
}

\author{
By \\ Moutaz M. Hegaze * \\ M. M, Abo El-Dahab **
}

\begin{abstract}
:
Composite materials are increasingly believed to be the materials of the future with potential for application in high performance structures. One of the reasons for that is the indication that composite materials have a rather good rating with regard to life time in fatigue. The present paper comprises theoretical and experimental research into the implementation of composite materials in structure applications. A finite element derivation was introduced based on Mindline theory to carry out stress analysis on different types of structures. Experimental measurements were carried out with two different types of composite materials, carbon/epoxy and glass/epoxy in order to obtain fatigue life diagrams ( $\mathrm{S} / \mathrm{N}$ diagrams) to be used for the fatigue damage assessment. Fatigue damage assessments were developed to predict the fatigue behavior of laminated structures based on damage by crack initiation under random load. A computer program was built based on the proposed finite element theory to carry out the previous analyses. The validation of the developed program for some analyses such as stress analysis and fatigue analysis was successfully achieved using a number of composite case studies.
\end{abstract}

\section{Keywords:}

Random load, composite materials, Fatigue damage, Stochastic Loading 
** Egyptian Armed Force, M. Sc.

\section{Introduction:}

Structural components are frequently subjected to complex time histories of stress for which the prevalent mode of failure is fatigue which consists of the formation (or initiation) of cracks and crack propagation under the action of varying loads. The failures of structures and mechanical engineering components have caused many injuries and much financial loss. In any event, failure due to fatigue is a fairly common mode. Perhaps it is therefore not so surprising that it has been estimated that at least $50 \%$ of failures which occur in engineering components can be attributed to fatigue.

Obviously, the loading anticipated on such structures is in sharp contrast to the usual laboratory fatigue test. It is essential in laboratory fatigue testing, of course, that tests are performed under known, carefully controlled conditions. However, real structures are subjected to service loadings which are often highly irregular and unpredictable. So there is a serious need for simple and reliable methods to predict such failures under loadings which are characterized as stochastic amplitudes. Unfortunately, fatigue of materials is still only partly understood. At the present, none of the existing methods provides demonstrated reliability. This challenges people to investigate fatigue problems and to improve design decisions involving fatigue.

In the design of structures subjected to random loading, such as bridges, towers, offshore structures, aircraft and vehicles subjected, respectively, to wind, earthquakes, sea waves, turbulence, rough roads and vibrations. A realistic description of the actual working stress can be obtained only in statistical way by modeling the corresponding irregular stress-time history as a random process. The theory of stochastic processes was introduced into the fields of civil engineering and mechanical engineering; it provides an appropriate mathematical tool for studying these erratic situations. The analysis of a stochastic process can either be done in the time or in the frequency domain [1,2].

The application of stochastic process theory has recently attracted many researchers. In recent years, a number of research workers in different countries have participated in the study of structural fatigue. Many researches[1-5] in this field studied this effect using samples subjected to random until failure, and failure is defined in many ways such as completely separation of the tested specimen into two pieces, or reaching a constrain value of strain or initiation of a crack or any way else.

The present paper comprises theoretical and experimental research into the implementation of composite materials in structure applications. A finite element derivation was carried out based on Mindline theory [6] and the residual force at each increment to carry extra load value and minimize the number of iterations for geometric nonlinearity case. Experimental measurements were carried out with two different types of composite materials, carbon/epoxy and glass/epoxy in order to obtain fatigue life diagrams ( $\mathrm{S} / \mathrm{N}$ diagrams) in fiber and matrix directions to be used for the fatigue damage assessment. Fatigue damage assessments were developed to predict the fatigue behavior of laminated structures based on damage by initiation under stochastic load. A computer program was built based on the proposed finite element theory in a module form to carry out the previous analyses. The proposed technique gives an automatic prediction to the number of cycle to failure for any structure subjected to stochastic load. 
This technique was validated through the validation of the proposed finite element results by published and finite element package (ANSYS) results.

\section{Finite-Element Derivation}

The proposed finite element derivation has been introduced based on Mindline theory and the residual force at each increment to carry extra load value and minimize the number of iterations for geometric nonlinearity case. Stress analysis parameters defined within plate are usually modeled in the finite element theory by a two-dimensional piecewise interpolation in terms of midplane parameters; together with analytical expressions in direction of thickness.

\subsection{Displacement, Strain \& Stress Components}

The displacement components at a general point may be resolved into in-plane displacements $\mathrm{u}, \mathrm{v}$. The displacement components can be expressed as,[7]

$$
\left.\begin{array}{l}
u(x, y, z)=u^{o}(x, y)+z \theta_{y}(x, y) \\
v(x, y, z)=v^{o}(x, y)-z \theta_{x}(x, y) \\
w(x, y, z)=\mathrm{w}(x, y)
\end{array}\right\}
$$

where $u^{o}, v^{o}$ represent displacement values at the midplane of the plate and $\theta_{x}, \theta_{y}$ are the average slope angles.

The degrees of freedom for a plate element with respect to its local axes, at its midplane, are given as follows:

$$
\begin{array}{ll}
\text { where } & \boldsymbol{\delta}_{o}=\left\{\begin{array}{llllllll}
u_{1} & v_{1} & u_{2} & v_{2} & \cdots & u_{n} & v_{n}
\end{array}\right\} \\
& \boldsymbol{\delta}_{b}=\left\{\begin{array}{llllllll}
w_{1} & \left(\theta_{x}\right)_{1} & \left(\theta_{y}\right)_{1} & \cdots & w_{n} & \left(\theta_{x}\right)_{n} & \left.\left(\theta_{y}\right)_{n}\right\}
\end{array}\right.
\end{array}
$$

For an n-node element the midplane displacement components and average slope angles at any point $(x, y)$ in the midplane of the plate can be interpolated as follows:

$$
\begin{array}{lll}
u^{o}(x, y)=\sum_{i=1}^{n} N_{i}(x, y) u_{i} & v^{o}(x, y)=\sum_{i=1}^{n} N_{i}(x, y) v_{i} & w(x, y)=\sum_{i=1}^{n} N_{i}(x, y) w_{i} \\
\theta_{x}(x, y)=\sum_{i=1}^{n} N_{i}(x, y)\left(\theta_{x}\right)_{i} & \theta_{y}(x, y)=\sum_{i=1}^{n} N_{i}(x, y)\left(\theta_{y}\right)_{i}
\end{array}
$$

where $u_{i}, v_{i}, w_{i},\left(\theta_{x}\right)_{i},\left(\theta_{y}\right)_{i}$ are values at node " i " and $N_{i}(x, y)$ represents Lagrangian shape functions [8].

For simplification of derivations, the strains are defined in terms of two separate vectors, X-y components vector $\varepsilon=\left\{\begin{array}{lll}\varepsilon_{x} & \varepsilon_{y} & \gamma_{x y}\end{array}\right\}^{T}$ and transverse shear vector $\gamma=\left\{\begin{array}{ll}\gamma_{x z} & \gamma_{y z}\end{array}\right\}^{T}$

Then according to strain displacement equations the vector of $x-y$ strain components can be expressed in terms of displacement components as follows, (without the effect of large deformation);

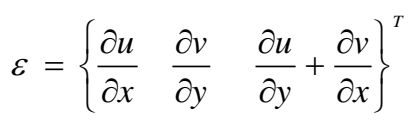

Using the displacement equations (1), the $x-y$ strain vector in equation (4) can be represented as follows:

$$
\varepsilon(x, y, z)=\varepsilon_{o}(x, y)-z \varepsilon_{b}(x, y)
$$

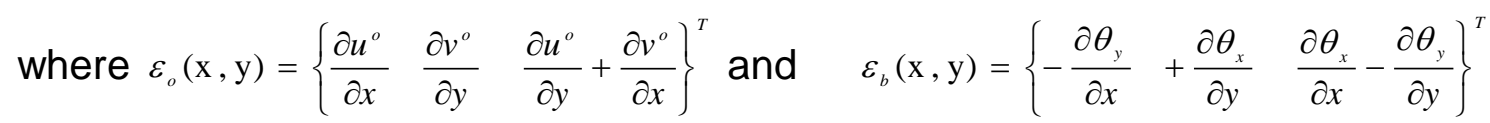


which can be represented as function of the shape function using equations (3) as;

$$
\boldsymbol{\varepsilon}(x, y, z)=\mathbf{B}_{o}(x, y) \boldsymbol{\delta}_{o}-z\left[\mathbf{B}_{b}(x, y) \boldsymbol{\delta}_{b}\right]
$$

Substituting from Equations ( $1 \& 3$ ) into Equation (4), then, The variation of the $x-y$ strain and the transverse shear strain vectors can be expressed as function of the shape function as follows:

$$
d \boldsymbol{\varepsilon}=\mathbf{B}_{o} d \boldsymbol{\delta}_{o}-z \mathbf{B}_{b} d \boldsymbol{\delta}_{b} \quad \text { and } \quad d \gamma(x, y, z)=f_{y}(z) \mathbf{B}_{\gamma}(x, y) d \boldsymbol{\delta}_{b}
$$

where $\mathbf{B}_{o}, \mathbf{B}_{b}$ and $\mathbf{B}_{\gamma}$ represent the shape function matrices, and $f_{\gamma}(z)=\frac{3}{2}\left(1-\frac{4 z^{2}}{h^{2}}\right)$

The relevant stress components at any point inside the $I^{\text {th }}$ layer of a composite layered plate can be represented in terms of two vectors defined as follows:

$$
\boldsymbol{\sigma}^{(i)}=\left\{\begin{array}{lll}
\sigma_{x} & \sigma_{y} & \tau_{x y}
\end{array}\right\}^{T} \quad \text { and } \quad \boldsymbol{\tau}^{(i)}=\left\{\begin{array}{ll}
\tau_{x z} & \tau_{y z}
\end{array}\right\}^{T}
$$

Using constitutive equations for the $\mathrm{I}^{\text {th }}$ layer, the stress vectors can be expressed in terms of strain vectors as follows:

$$
\boldsymbol{\tau}^{(i)}=\boldsymbol{\mu}^{(i)} \boldsymbol{\gamma} \text { and } \boldsymbol{\sigma}^{(i)}=\mathbf{D}^{(i)} \boldsymbol{\varepsilon}
$$

where $\boldsymbol{\mu}^{(i)}$ and $\mathbf{D}^{(t)}$ are the stiffness matrices, which are function of the material properties of layer $\mathrm{I}^{\text {th }}$, with respect to transverse and $\mathrm{x}$-y stress components.

\subsection{Variation of Strain Energy}

The variation of strain energy density at any point inside the $I^{\text {th }}$ layer of the plate can be expressed as follows:

$$
d \bar{U}^{(i)}=d \mathbf{\delta}_{b}^{t} \mathbf{B}_{\gamma}^{t} f_{\gamma}(z) \boldsymbol{\tau}^{(i)}+\left[d \mathbf{\delta}_{o}^{t}\left(\mathbf{B}_{o}^{t}\right)-z\left(d \mathbf{\delta}_{b}^{t}\left(\mathbf{B}_{b}^{t}\right)\right)\right] \boldsymbol{\sigma}^{(i)}
$$

By integrating over the layers with respect to $z$, then it can be deduced that:

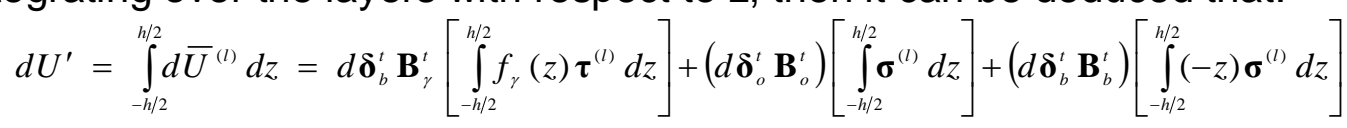

which can be written in terms of integrated stress vectors as follows:

$$
d U^{\prime}=d \mathbf{\delta}_{b}^{\prime} \mathbf{B}_{y}^{\prime} \tilde{\boldsymbol{\tau}}+\left(d \mathbf{\delta}_{o}^{\prime} \mathbf{B}_{o}^{t}\right) \boldsymbol{\sigma}_{o}+\left(d \mathbf{\delta}_{b}^{\prime} \mathbf{B}_{b}^{t}\right) \boldsymbol{\sigma}_{1}
$$

where the Integrated $x-y$ and transverse shear stresses can be defined as;

$$
\tilde{\boldsymbol{\tau}}=\boldsymbol{\mu}_{\gamma \gamma} \hat{\boldsymbol{\gamma}} \equiv \boldsymbol{\mu}_{\gamma \gamma} \mathbf{B}_{\gamma} \boldsymbol{\delta}_{b} \quad \text { and } \quad \boldsymbol{\sigma}_{n}=(-1)^{n}\left[\mathbf{D}_{n}\left(\boldsymbol{\varepsilon}_{o}\right)-\mathbf{D}_{n+1}\left(\boldsymbol{\varepsilon}_{b}\right)\right]
$$

where $\quad \boldsymbol{\mu}_{\gamma \gamma}=\int_{-h / 2}^{h / 2} f_{\gamma}^{2}(z) \boldsymbol{\mu}^{(i)} d z \equiv \int_{-h / 2}^{h / 2} \frac{9}{4}\left(1-\frac{4 z^{2}}{h^{2}}\right)^{2} \boldsymbol{\mu}^{(l)} d z \quad$ and $\quad \mathbf{D}_{n}=\int_{-h / 2}^{h / 2} z^{n} \mathbf{D}^{(l)} d z$

\subsection{Generalized Equations of Equilibrium}

The wok done by actual external loads can be expressed in terms of equivalent nodal loading represented with the following vector:

$$
\mathbf{F} \equiv\left[\begin{array}{ll}
F_{o} & F_{b}
\end{array}\right]^{r}
$$

Hence the variation of the work done by external loads is equal to the variation of the work done by the corresponding equivalent nodal loading, i.e.

$$
d W=d \mathbf{\delta}_{o}^{\prime} \mathbf{F}_{o}+d \mathbf{\delta}_{b}^{\prime} \mathbf{F}_{b}
$$

The variation of the strain energy for the whole domain, with a finite element mesh containing Ne elements, is given by:

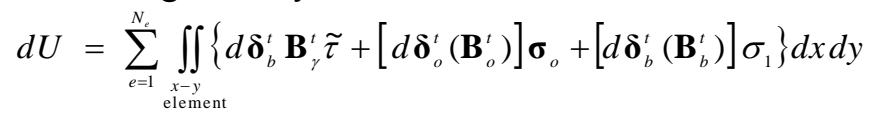

Using the principle of virtual work, then

$$
d U-d W=0
$$


Substituting from Equations (9) and (10) into (11), then it can be shown that:

$$
\sum_{\substack{e=1 \\ N_{c}}}^{N_{x}} \int_{\text {element }}\left\{d \boldsymbol{\delta}_{b}^{t} \mathbf{B}_{\gamma}^{t} \tilde{\tau}+\left[d \boldsymbol{\delta}_{o}^{t}\left(\mathbf{B}_{o}^{t}\right)\right] \boldsymbol{\sigma}_{o}+\left[d \boldsymbol{\delta}_{b}^{t}\left(\mathbf{B}_{b}^{t}\right)\right] \sigma_{1}\right\} d x d y-\left[d \boldsymbol{\delta}_{o}^{t} \mathbf{F}_{o}+d \boldsymbol{\delta}_{b}^{t} \mathbf{F}_{b}\right]=0
$$

Since $d \mathbf{\delta}_{o}, d \mathbf{\delta}_{b}$ represent arbitrary parameters, then their coefficients in equation (12) will lead to residual vectors as:

$$
\mathbf{R}_{o}=\mathbf{F}_{o}-\sum_{e=1}^{N_{e}} \iint_{e}\left[\mathbf{B}_{o}^{t} \boldsymbol{\sigma}_{o}\right] d x d y \quad \text { and } \quad \mathbf{R}_{b}=\mathbf{F}_{b}-\sum_{e=1}^{N_{e}} \iint_{e}\left[\mathbf{B}_{\gamma}^{t} \tilde{\boldsymbol{\tau}}+\mathbf{B}_{b}^{t} \boldsymbol{\sigma}_{1}\right] d x d y
$$

\subsection{Linearization of Equations of Equilibrium}

To make the residual vectors vanish we assume (to restore equilibrium):

$$
\boldsymbol{\delta}_{\text {new }}=\boldsymbol{\delta}_{\text {old }}+\Delta \boldsymbol{\delta} \quad \boldsymbol{\sigma}_{\text {new }}^{(l)}=\boldsymbol{\sigma}_{\text {old }}^{(l)}+\Delta \boldsymbol{\sigma}
$$

such that $\quad \mathbf{R}_{o}, \mathbf{R}_{b}$ in equation (13) tends to zero, then they can be reduced to;

$\sum_{e=1}^{N} \iiint_{e}\left\{\left[\mathbf{B}_{o}^{t}\right] \Delta \boldsymbol{\sigma}^{(i)}\right\} d x d y d z=\mathbf{R}_{o} \quad \sum_{e=1}^{N} \iiint_{e}\left\{\mathbf{B}_{\gamma}^{t} f_{\gamma}(z) \Delta \boldsymbol{\tau}^{(i)}+\left[\mathbf{B}_{b}^{t}\right]\left(-z \Delta \boldsymbol{\sigma}^{(i)}\right)\right\} d x d y d z=\mathbf{R}_{b}$

which can be written as follows:

$\sum_{e=1}^{N}\left[\left(\mathbf{R}_{o}^{c}\right)_{\Delta s}\right]=\mathbf{R}_{o} \quad \sum_{e=1}^{N}\left[\left(\mathbf{R}_{b}^{e}\right)_{\Delta s}\right]=\mathbf{R}_{b}$

where, with the use of non-linear $B$ and $D$ matrices:
$\left(\mathbf{R}_{o}^{e}\right)_{\Delta \sigma}=\mathbf{K}_{o o} \Delta \boldsymbol{\delta}_{o}+\mathbf{K}_{o b} \Delta \boldsymbol{\delta}_{b}$
$\left(\mathbf{R}_{b}^{c}\right)_{\Delta \sigma}=\mathbf{K}_{b o} \Delta \boldsymbol{\delta}_{o}+\mathbf{K}_{b b} \Delta \boldsymbol{\delta}_{b}+\mathbf{K}_{w} \Delta \boldsymbol{\delta}_{b}$

where

$$
\begin{aligned}
& \mathbf{K}_{o o}=\iint\left[\mathbf{B}_{o}^{t} \mathbf{D}_{o} \mathbf{B}_{o}\right] d x d y \\
& \mathbf{K}_{o b}=\iint\left[\mathbf{B}_{o}^{t} \mathbf{D}_{1} \mathbf{B}_{b}\right] d x d y \\
& \mathbf{K}_{b o}=\iint\left[\mathbf{B}_{b}^{t} \mathbf{D}_{1} \mathbf{B}_{o}\right] d x d y \equiv \mathbf{K}_{o b}^{t} \\
& \mathbf{K}_{r y}=\iint \mathbf{B}_{\gamma}^{t} \boldsymbol{\mu}_{r} \mathbf{B}_{\gamma} d x d y \\
& \mathbf{K}_{b b}=\iint\left[\mathbf{B}_{b}^{t} \mathbf{D}_{2} \mathbf{B}_{b}\right] d x d y
\end{aligned}
$$

where the element stiffness matrices are defined as follow:

$$
\mathbf{K}=\left[\begin{array}{cc}
\mathbf{K}_{o o} & \mathbf{K}_{o b} \\
\mathbf{K}_{b o} & \mathbf{K}_{b b}+\mathbf{K}_{w}
\end{array}\right]
$$

\section{Experimental setup:}

Two types of fibre reinforced materials have been used in this work, carbon/epoxy (Fiberite 977-2 toughened epoxy resin) by Cytec Fiberite Ltd. and glass/epoxy (Fibredux 914G-E-5-30\%) by CIBA-GEIGY Ltd with materiald properties as shown in Table 1,

Table 1. Material properties of Carbon/epoxy and Glass/epoxy lamina

\begin{tabular}{|l|c|c|}
\hline \multicolumn{1}{|c|}{ Parameter } & Carbon/epoxy & Glass/epoxy \\
\hline Longitudinal Modulus $\mathrm{E}_{1},[\mathrm{GPa}]$ & 128.0 & 35.0 \\
\hline Transverse Modulus $\mathrm{E}_{2},[\mathrm{GPa}]$ & 11.0 & 8.22 \\
\hline $\begin{array}{l}\text { Shear Modulus in } \mathrm{x}-\mathrm{y} \text { plane } \mathrm{G}_{12}, \\
\text { [GPa] }\end{array}$ & 4.48 & 4.1 \\
\hline $\begin{array}{l}\text { Shear Modulus in y-z plane } \mathrm{G}_{23}, \\
{[\mathrm{GPa}]}\end{array}$ & 4.48 & 4.1 \\
\hline $\begin{array}{l}\text { Shear Modulus in z-x plane } \mathrm{G}_{31}, \\
\text { [GPa] }\end{array}$ & 4.48 & 4.1 \\
\hline In-plane Poisson's Ratio $\mathrm{v}_{12}$ & 0.25 & 0.26 \\
\hline Thickness of each layer, [mm] & 0.25 & 0.15 \\
\hline Density $\rho,\left[\mathrm{kg} / \mathrm{m}^{3}\right.$ ] & 1500 & 2000 \\
\hline
\end{tabular}


Some experimental works are presented in this paper to provide fatigue life curves (S-N diagrams in fiber and matrix directions) and to create a stochastic loads for the two types of laminates mentioned earlier. The materials are prepared in panel forms with different stacking sequences based on the type of tests.

\subsection{Fatigue test}

The fatigue test specimens are straight-sided coupons of constant cross section as described in ASTM D3039-76 (1989). Recommended thickness is 0.5 to $2.5 \mathrm{~mm}(0.02$ to $0.1 \mathrm{in})$ usually six plies $\left[0^{\circ}\right]_{6}$ for the longitudinal specimen to predict the $\mathrm{S}-\mathrm{N}$ diagram in fiber direction and at least eight plies $\left[{ }^{\circ}\right]_{8}$ for the transverse one to predict the S-N diagram in matrix direction. However, it was necessary to modify the configuration in order to obtain acceptable values (to ensure failure in the gauge area and not within the area in contact with the test grips).

The main purpose of this test is to provide the finite element program by the fatigue life diagrams (S-N diagrams in fiber and matrix directions) to use in the prediction of the number of cycle to faultier as it will illustrate later.

The type of specimens used depends on the objective of the test program, the type of equipment capacity and the form in which the material is available. However, the design must meet certain general criteria, [9]:

- Failure occurs in the test section.

- The absolute maximum stress should not be less than $25 \%$ of the machine operating stress range being used and the absolute minimum stress should not be less than $2.5 \%$ of the machine operating range being used.

The measurement process is repeated several times at each level of stress to obtain a more accurate $\mathrm{S} / \mathrm{N}$ diagram based on average value. The experimental fatigue test analyses have been carried out as explained to obtain the fatigue life diagrams for carbon/epoxy and glass/epoxy materials. The S-N diagrams for carbon/epoxy and glass/epoxy are shown in Figures $1, \& 2$, respectively.

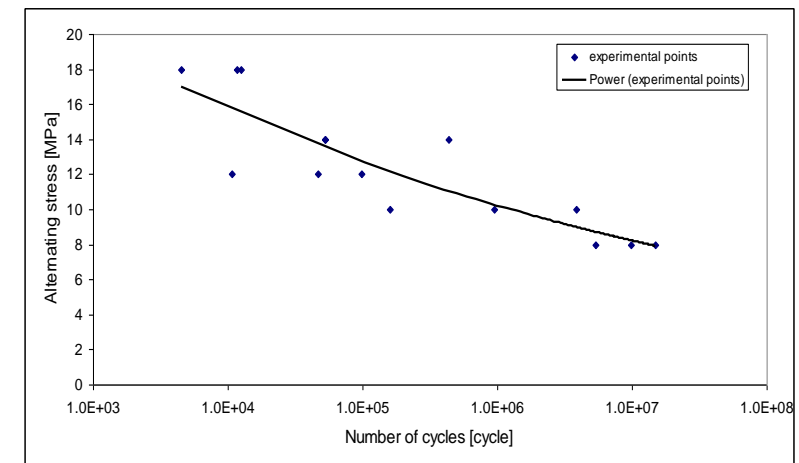

Figure 1. S-N diagram for carbon/epoxy

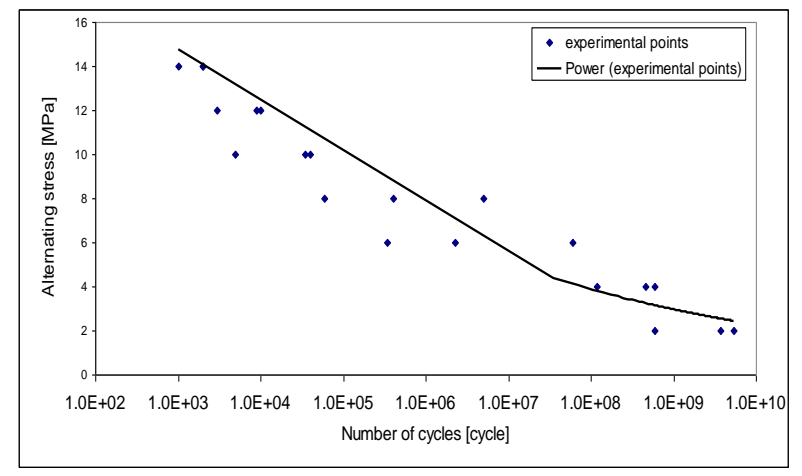

Figure 2. S-N diagram for glass/epoxy 


\subsection{Random test \& Cycle counting method (rainflow)}

The specimens were fixed to have a base excitation; the signal was generated by an analyzer and signal generator which were connected to the shaker. The force was measured with a force transducer placed between shaker and fixture as an input signal for the analyzer. The response was measured by an accelerometer which gives feed back to the analyzer. This analyzer can give the input and output signal characteristics after excitation with an internally generated signal of random load. The analyzer can be connected to a computer with special software so that all data are saved on a disc to be reviewed. Figure 3, shows a schematic diagram for the system of measurements and the overall system

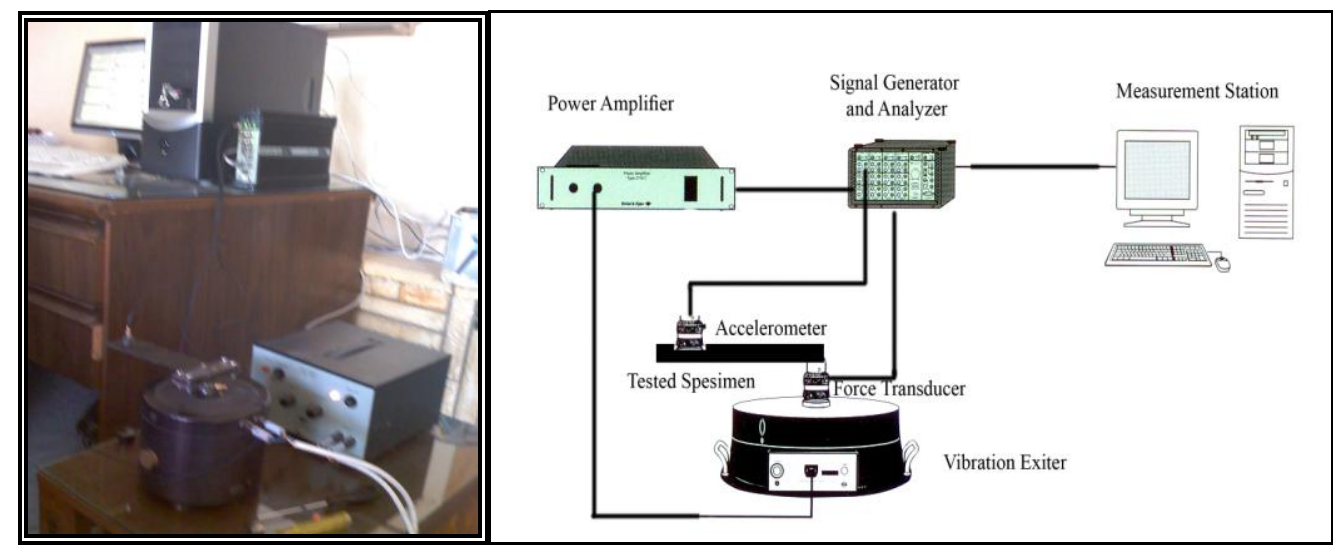

Figure 3. a schematic diagram for the system of measurements and the overall system.

Figure 4 shows the applied random force on Carbon / Epoxy and Glass/Epoxy (160x40) $\mathrm{mm}$ specimens (sensor placed at $2 \mathrm{~cm}$ from the end). The data was recorded during 0.015 second in 512 data point.

Cycle counting methods are devised in order that the effect of irregular load histories can be compared with the damage resulting from regularly repeated simple load cycles as typified by S-N diagrams. The application of the damage rule requires that we know the mean and amplitude of stress to which the damaging event should be compared.

$$
D=\sum_{\mathrm{i}=1}^{\mathrm{n}} \frac{1}{\mathrm{~N}_{\mathrm{i}}}
$$

Where $\mathrm{N}_{\mathrm{i}}$ represent the number of cycle to failure for $\mathrm{i}$ group of cycles and $\mathrm{n}$ represent the number of group of cycles. The rainflow method is generally accepted as the best available for the identification of ranges in a stress history [9]. The range is counted when the stress first returns to the level of a previous peak that has not been included in a range before. Another visualization of cycle identification is picturing the cycles identified by the depth of water caught by the shape of the signal if it were a series of reservoirs. The rainflow method, like the peak method, is easily programmed and, given a history of peaks and valleys, quickly identifies the ranges of the stress history. 


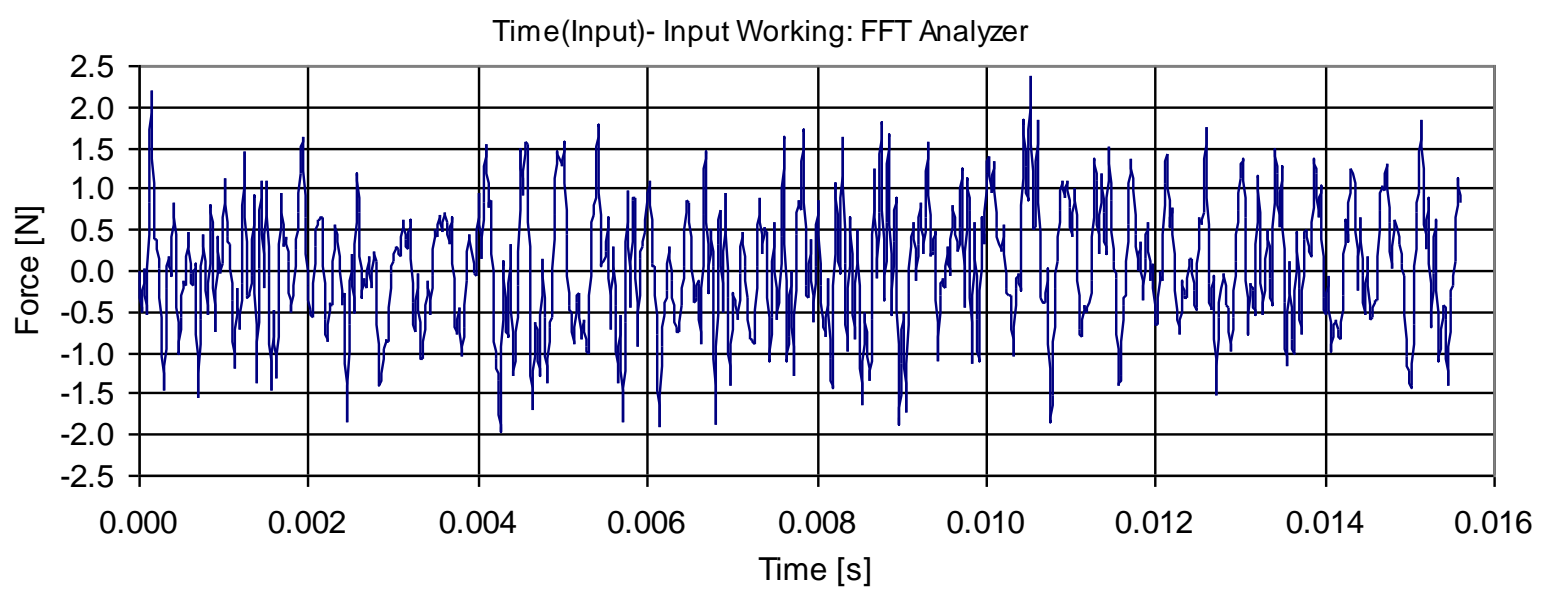

Figure 4. Force applied on the Carbon / Epoxy and Glass/Epoxy $(160 \times 40) \mathrm{mm}$ specimens

\section{Fatigue damage analysis by Crack initiation:}

The initiation of a crack does not mean a complete failure of a structure but it gives an indication of the start of the fatigue process that leads to complete failure. The fatigue by crack initiation does not lead to complete failure of a structure before it is detected and repaired. Special components in some industrial applications have to be repaired or replaced when fatigue crack initiation is detected, especially in aerospace applications. An assessment of fatigue damage by initiation is studied in this paper to simulate the fatigue behavior of unidirectional composite laminates under stochastic load conditions and to predict the fatigue life of these laminates under this type of load.

This study is carried out to establish a technique to use experimental data from unidirectional ply under uniaxial cyclic load to simulate the fatigue behavior of laminates, which have the same constituents of that ply. On the other hand, this technique can give an early warning on the initiation of the fatigue damage and to estimate the number of cycles needed for the crack to start or grow in general applications under general load conditions.

\subsection{Fatigue analysis}

The fatigue analysis will be based upon experimental data in fiber and matrix materials of the composite and the fatigue theory of metals. The experimental data needed for this model are the stress-life $\mathrm{S} / \mathrm{N}$ curves for fiber and matrix materials. However, if the specimen does not fail, the applied load creates a stress under the endurance limit of the fiber and matrix of the composite laminate.

\subsection{The fatigue damage by initiation assessment in steps}

Consider that it is required to investigate the fatigue behavior of a composite laminate under general applied cyclic load. Since no actual cracks are assumed, linear stress analysis can be considered for this type of fatigue damage.

\section{Step 1: preparations and basic assumptions}

The fatigue load is applied uniformly with frequency $f[\mathrm{~Hz}]$. The maximum and minimum load ratios $\alpha_{\max }$ and $\alpha_{\min }$ are defined to represent the maximum and minimum load, 
respectively.

The actual load is represented by a constant load vector $\hat{\hat{F}}$ and the load ratio $\alpha(t)$ as follows;

$$
\underline{F}(\mathrm{t})=\alpha(\mathrm{t}) \underline{\hat{\mathrm{F}}}
$$

\section{Step 2: stress analysis using finite element method}

Finite element method is used to analyze and evaluate the stresses and strains at any node (i) with respect to local coordinate system. A suitable transformation process is used to transfer the stresses $\underline{\sigma}_{i}$ to the material coordinate system in the fiber and matrix directions. The actual stress value at any time and at any node (i) can be defined similar to the force vector in equation (16) as follows:

$$
\underline{\sigma}_{\mathrm{i}}(\mathrm{t})=\alpha(\mathrm{t}) \underline{\sigma}_{i}
$$

The alternating and mean stresses at node (i) can be represented as follows:

$$
\left.\left.\underline{\sigma}_{\mathrm{a}}\right)_{\mathrm{i}}=\alpha_{\mathrm{a}} \underline{\sigma}_{i} \quad \text { and } \quad \underline{\sigma}_{\mathrm{m}}\right)_{\mathrm{i}}=\alpha_{\mathrm{m}} \underline{\sigma}_{i}
$$

Hence, the critical values of alternating $\left.\underline{\sigma}_{\mathrm{a}}\right)_{\mathrm{cr}}$ and mean $\left.\underline{\sigma}_{\mathrm{m}}\right)_{\mathrm{cr}}$ stress can be obtained at critical node points.

\section{Step 3: Stress checking}

Before the fatigue analysis takes place, stress checking has to be completed. The stress checking is to ensure that the stress values occur between the endurance and ultimate stress limits of the material and satisfy the following condition:

$$
\sigma_{u}>\left(\sigma_{\mathrm{a}}\right)_{c r}>\sigma_{\mathrm{e}}
$$

where $\sigma_{u}$ is the ultimate stress of the material and $\sigma_{e}$ is the endurance limit.

\section{Step 4: Fatigue analysis}

As a first approximation, Goodman relationship, which is an empirical relationship between the applied stress and the fatigue stress, [12], is employed and can be defined as follows:

$$
\frac{\sigma_{a}}{\sigma_{N}}+\frac{\sigma_{\mathrm{m}}}{\sigma_{\mathrm{u}}}=1
$$

Applying equation (18) in the fiber and matrix directions separately for the failed elements as follows:

$$
\begin{aligned}
& \frac{\left(\sigma_{a, f}\right)_{c r}}{\sigma_{N f}}+\frac{\left(\sigma_{\mathrm{m}, \mathrm{f}}\right)_{c r}}{\sigma_{\mathrm{uf}}}=1 \\
& \frac{\left(\sigma_{a, m}\right)_{c r}}{\sigma_{N m}}+\frac{\left(\sigma_{\mathrm{m}, \mathrm{m}}\right)_{c r}}{\sigma_{\mathrm{um}}}=1
\end{aligned}
$$

where the subscripts $f$ and $m$ mean fiber and matrix, respectively.

The fatigue stresses of the fiber $\sigma_{N f}$ and matrix $\sigma_{N m}$ can be obtained by applying equations (19), respectively. The corresponding number of cycles to fatigue of fiber $N_{f}$ and matrix $N_{m}$ can be obtained by using S/N diagram for fiber and matrix. The final number of cycles to fatigue of the composite laminate is the minimum value of the number of cycles to failure for fiber $N_{f}$ and matrix $N_{m}$. It is expected that the failure is caused by matrix cracking mode, which is considered the dominant damage mode for fatigue failure of composite materials 


\section{5 validation and numerical case studies}

The proposed technique can be validated through several steps; firstly, the validation of the finite element derivation by comparing its results with published results or with a known finite element package (ANSYS), secondly, check the accuracy of measurements of the S-N diagrams by repeat the measurements different times at the same level of stress, finally, good prediction of the random load and check its transformations from original form to the rainflow form. A computer program was developed based on the finite element theory presented earlier to carry out some analyses based on that theory.

The program can be used to analyze shell or plate stress analysis applications, which may be linear. The main types of analysis which are carried out in this program are linear stress analysis and fatigue damage analysis (damage by crack initiation). The program was coded in Fortran 90 by means of Digital Visual FORTRAN V.6 under Microsoft Windows.

\subsection{Stress validation and mesh convergence}

Different stress analyses have been carried out to ensure and validate the accuracy of the derived finite element and its implementation in the programming. One of the important problems in finite element analysis is the selection of the optimum finite element mesh so as to obtain accurate results with a reasonable number of elements. In this section, quadrilateral 4-node elements and triangular 3-node element have been used for the analyses with different meshes to validate the linear analysis and to study the mesh convergence.

\section{Simply supported square plate (SSSP)}

The square plate has been used before in many publications [10]. Consider a laminate square plate with 8 layers [0o/45o/-45o/90o]s and the following material properties:

$\mathrm{E} 1=40 \mathrm{E} 2, \mathrm{G} 12=0.6 \mathrm{E} 2, \mathrm{G} 23=0.5 \mathrm{E} 2, \mathrm{v} 12=0.25$, and it is assumed that $\mathrm{G} 13=\mathrm{G} 12, \mathrm{v} 13$ $=\mathrm{v} 12$ and $\rho=1$

The plate is subjected to two different types of transverse loadings, sinusoidal load, and uniform load with intensity $q_{o}$ to test the package with different types of loads where the load has a shear force per unit area q represented as:

$$
\text { Uniform load } q(x, y)=\mathrm{q}_{\mathrm{o}} \quad \text { and } \quad \text { Sinusoidal load } q(x, y)=\mathrm{q}_{\mathrm{o}} \sin \frac{\pi \mathrm{x}}{\mathrm{L}}
$$

The plate is simply supported at all the four edges and the central deflections are represented by a non-dimensional deflection parameter $\bar{W}$ as follows:

$$
\bar{W}=\frac{\mathrm{w}_{\mathrm{c}} \mathrm{E}_{2} \mathrm{t}^{3}}{\mathrm{q}_{\mathrm{o}} \mathrm{L}^{4}} \times 10^{2}
$$

where wc is the central deflection, $L$ and $t$ are the side length and the thickness of the plate, respectively.

Due to the symmetry of the plate, a quarter-plate mesh is used. Two different types of mesh have been applied with this study, a coarse mesh (c.) and a fine mesh (f.) as shown in Figures 5 and 6 to study the mesh convergence 


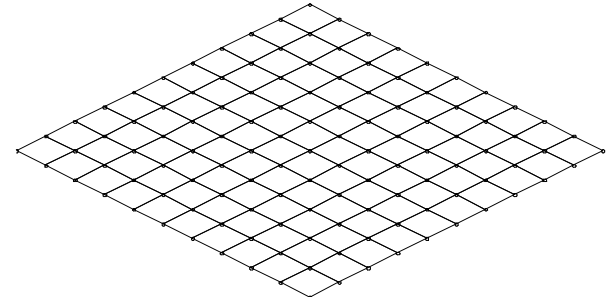

a- Coarse mesh (c.) with 100 4-node elements

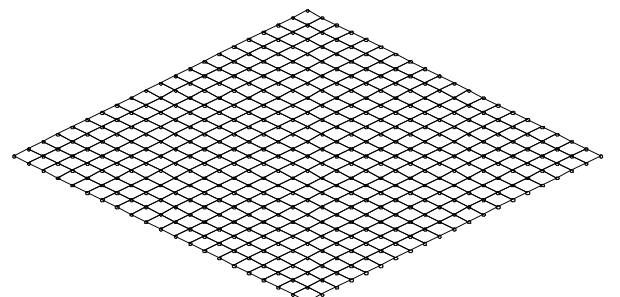

b- Fine mesh (f.) with 400 4-node elements

Figure 5. Meshes of a square plate with 4-node quadrilateral elements.
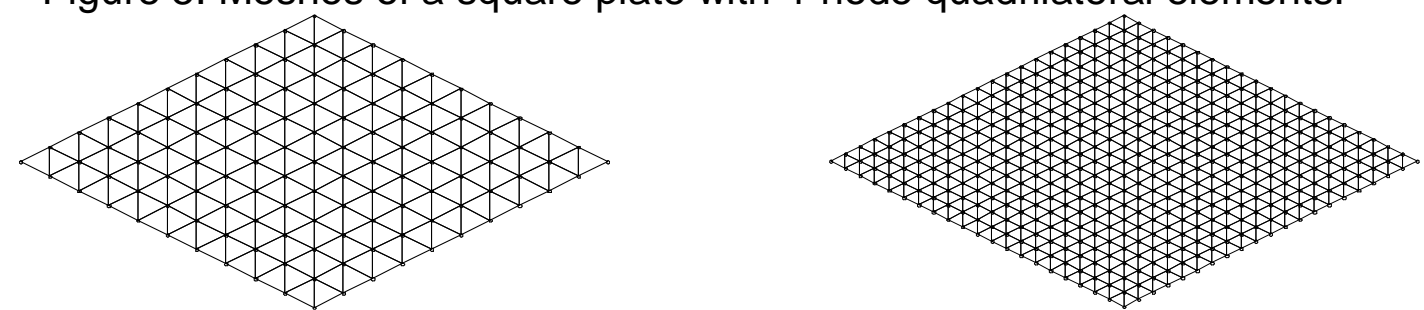
a- Coarse mesh
elements.
(c.) with 200 3-node
b- Fine mesh
(f.) with 800 3-node elements.

Figure 6. Meshes of a square plate with 3-node triangle element.

Table 2 summarize the results by comparing the non-dimensional deflection parameter $\bar{W}$ of the simply supported square plate under uniform load using different elements with the published results of Phan \& Reddy [10] and the results obtained with the ANSYS commercial package. It is clear from the results presented in the tables that all elements give good results and the two types of meshes providing very close results to each other

Table 2. Non dimensional deflection parameter of SSSP subjected to uniform load

\begin{tabular}{|c|c|c|c|c|c|c|c|}
\hline L/t & $\begin{array}{c}\text { Phan \& } \\
\text { Reddy } \\
(1985)\end{array}$ & $\begin{array}{c}\text { ANSYS } \\
\text { Shell 99 } \\
\text { (c.) }\end{array}$ & $\begin{array}{c}\text { ANSYS } \\
\text { Shell 99 } \\
\text { (f.) }\end{array}$ & $\begin{array}{c}\text { 4-Node } \\
\text { Element } \\
\text { (c.) }\end{array}$ & $\begin{array}{c}\text { 4-Node } \\
\text { Element } \\
\text { (f.) }\end{array}$ & $\begin{array}{c}\text { 3-Node } \\
\text { Element } \\
\text { (c.) }\end{array}$ & $\begin{array}{c}\text { 3-Node } \\
\text { Element } \\
\text { (f.) }\end{array}$ \\
\hline 4 & 1.634 & 1.672 & 1.669 & 1.794 & 1.806 & 1.790 & 1.805 \\
\hline 10 & 0.590 & 0.644 & 0.644 & 0.686 & 0.679 & 0.669 & 0.676 \\
\hline 20 & 0.434 & 0.470 & 0.470 & 0.496 & 0.486 & 0.477 & 0.480 \\
\hline 50 & 0.386 & 0.404 & 0.405 & 0.423 & 0.413 & 0.405 & 0.407 \\
\hline 100 & 0.377 & 0.387 & 0.389 & 0.405 & 0.395 & 0.389 & 0.390 \\
\hline
\end{tabular}

Figure 7 show the non dimensional deflection parameter $\bar{W}$ of the simply supported square plate under sinusoidal load using different elements as a function of the span-tothickness ratio. As seen in the figures, the program results, which were obtained using the coarse mesh, have a good agreement with the corresponding results by ANSYS package. While the published results of Phan \& Reddy [10] have a certain shift all over the span-tothickness ratio range which is due to the approximations employed in their theory. 

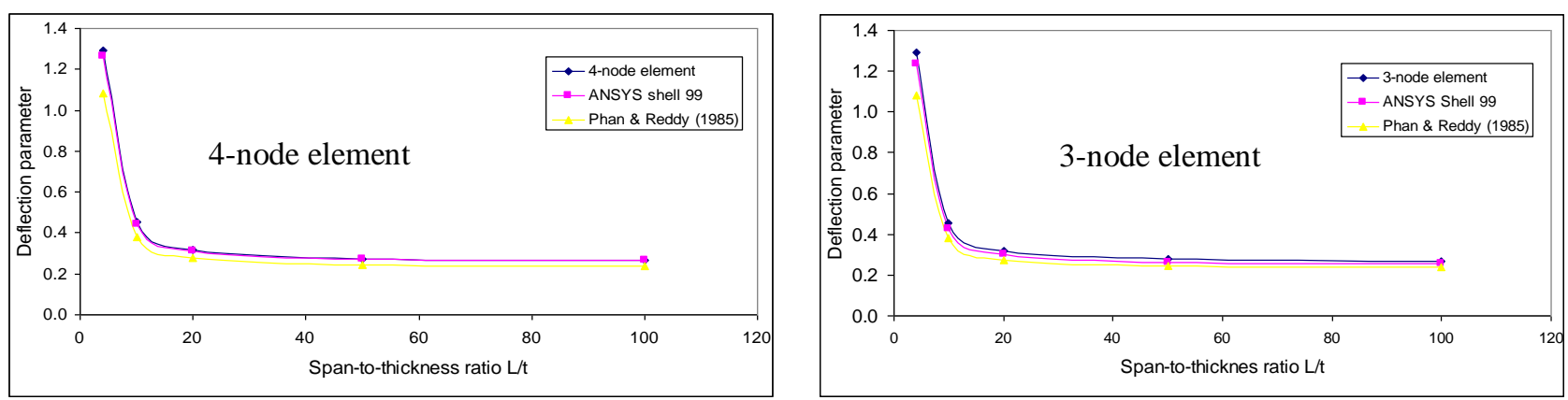

Figure 7 Non dimensional deflection parameter versus span-to-thickness ratio of SSSP subjected to sinusoidal load

It is clear from the figures that the finite element programming give results that compare well with ANSYS results and the published results by Phan \& Reddy [10] for the different types of loads. It can also be seen from the shown results that the two types of meshes provide very close results. Hence, it was decided to use only the 4-node element with coarse mesh for further validation or analyses.

\subsection{Fatigue damage analysis by initiation}

The previous section illustrates the validation of the finite element programming from several points of view (type of analysis, type of loads, mesh selection and span to thickness ratio). The finite element program is used in this section for the fatigue analysis by crack initiation in composite materials, which was discussed earlier.

Two symmetric 12-layer $\left[0^{\circ} / 90^{\circ} / 45^{\circ} / 0^{\circ} /-45^{\circ} / 90^{\circ}\right]_{s}$ composite plates $(160 \times 40)$ have been used in this analysis, with two different types of materials, carbon/epoxy and glass/epoxy, which were also used in the experimental work.

The plates were subjected to the random load shown in Figure 4 which was generated form the analyzed. This load can be sorted automatically after its generation using the sorting module in the proposed program to identify the maximum and minimum applied load as shown in Figure 8 and to convert the applied load to the rainflow form. The data was recorded and reduced from 512 data points to 298 data points (maximum or minimum).

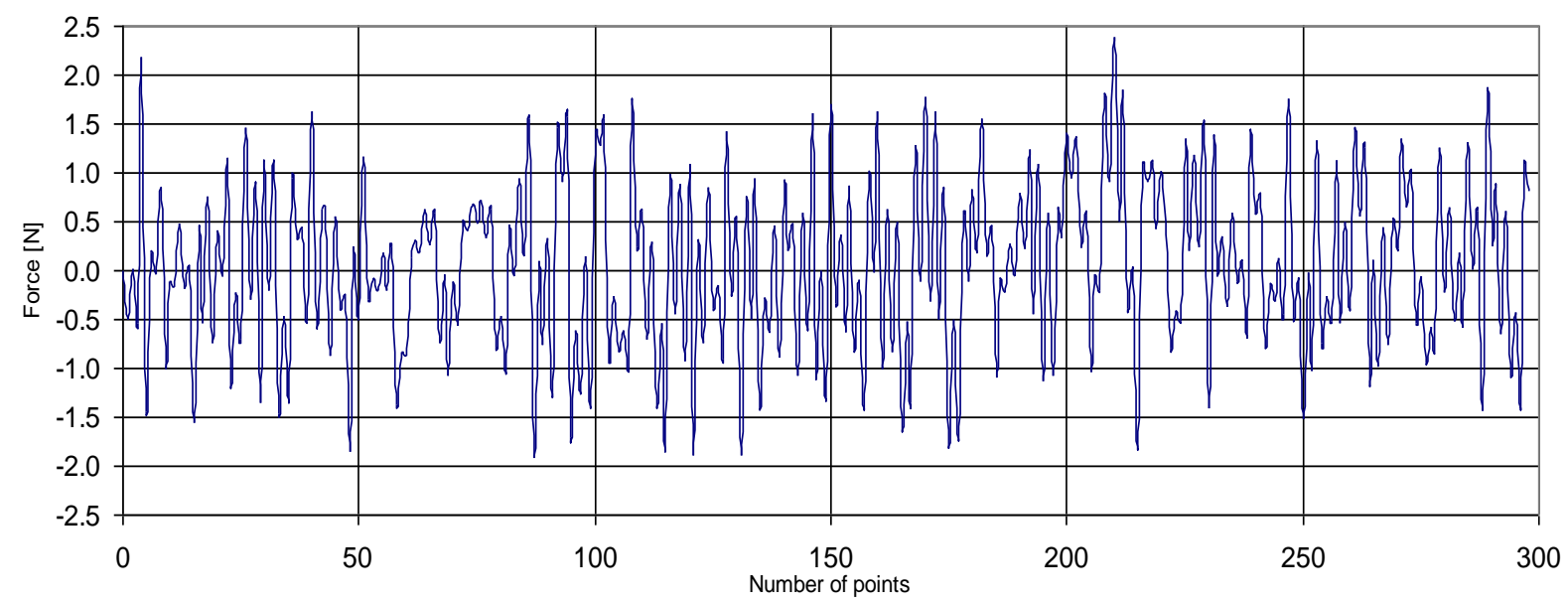

Figure 8. Applied random load in rainflow form

This random load can be rearranged using the rainflow method to obtain 147 groups of cyclic load each one of them has mean and amplitude values as shown in Figure 9. 


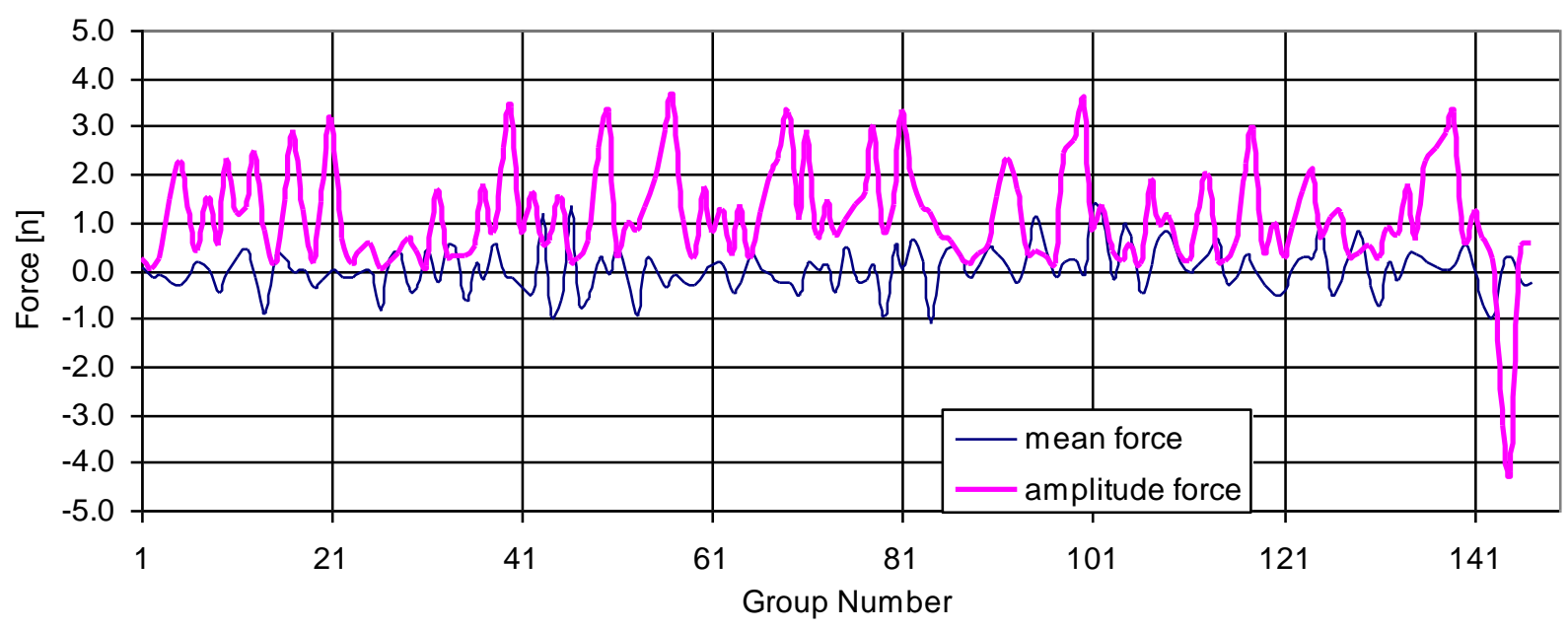

Figure 9. Mean and amplitude values of the random load

The stress analyses presented in this part are carried out under the effect of unit load. The maximum and minimum stresses were defined and obtained based on load ratios $\alpha_{\max }$ and $a_{\min }$ for each load cycle as indicated in Table 3. Apply Palmgren-Miner law in equation (15) for the rainflow cycle counting to calculate the damage then the number of cycle to failure. Table 4 shows the number of cycle to failure for carbon/epoxy and glase/epoxy.

Table 4. Number of cycles to failure for carbon and Glass/epoxy.

\begin{tabular}{|c|c|c|c|c|}
\hline & Carbon/epoxy & Glass/epoxy \\
\hline \multirow{4}{*}{ 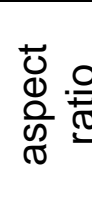 } & \multirow[t]{2}{*}{$\mathrm{L} / \mathrm{b}=1$} & Fiber & $4.4 \times 10^{11}$ & $14.4 \times 10^{17}$ \\
\hline & & Matrix & $7.21 \times 10^{6}$ & $14.1 \times 10^{5}$ \\
\hline & \multirow{2}{*}{$\mathrm{L} / \mathrm{b}=2$} & Fiber & $5.56 \times 10^{11}$ & $18.34 \times 10^{17}$ \\
\hline & & Matrix & $6.67 \times 10^{6}$ & $14.45 \times 10^{5}$ \\
\hline
\end{tabular}

\section{Conclusion:}

Two specimens, carbon/epoxy and glass/epoxy have been subjected to random load. The output measurements are recorded and plotted as a time history. Specimens were fixed from one end and free from the other end while sensors were located in two different places $2 \mathrm{~cm}$ and $7 \mathrm{~cm}$ from the free end. The output data were recoded and analyzed by the vibration analyzer to get the response.

One of the main objectives of this work was to include the fatigue damage assessment with the finite element analysis. A versatile and efficient finite element formulation of plate element is employed to determine the stiffness and mass matrices based on Mindline's theory. The fatigue damage assessment, depending on the case study, is presented with fatigue damage by initiation under random load.

The proposed technique has been validated through several steps; firstly, the validation of the finite element derivation by comparing its results with published results or with a known finite element package (ANSYS), secondly, the accuracy of measurements of the $\mathrm{S}-\mathrm{N}$ diagrams has been checked by repeat the measurements different times at the same level of stress, finally, good prediction of the random load and check its transformations from original form to the rainflow form have been done. 
A finite element derivation was carried out based on Mindline theory and the residual force at each increment to carry extra load value and minimize the number of iterations for geometric nonlinearity case. Experimental measurements were carried out with two different types of composite materials, carbon/epoxy and glass/epoxy in order to obtain fatigue life diagrams ( $\mathrm{S} / \mathrm{N}$ diagrams) in fiber and matrix directions to be used for the fatigue damage assessment. Fatigue damage assessments were developed to predict the fatigue behavior of laminated structures based on damage by initiation under stochastic load.

A computer program was built based on the proposed finite element theory in a module form to carry out the previous analyses. The proposed technique gives an automatic prediction to the number of cycle to failure for any structure subjected to stochastic load. This technique was validated through the validation of the proposed finite element results by published and finite element package (ANSYS) results.

The results have been recognized for different aspect ratios and with different types of materials and some conclusions are found as follows:

- Maximum stresses at glass/epoxy case are higher than at carbon/epoxy case for different aspect ratios.

- The maximum fiber stresses observed in layers with $0^{\circ}$ of fiber orientation angle.

- The maximum matrix stresses observed in layers with $90^{\circ}$ of fiber orientation angle.

-The formulation and implementation of an efficient automatic algorithm for fatigue life assessment of composites by fatigue damage by crack initiation, which leads to a considerable saving in user efforts and computer resources.

\section{References:}

B. R. E. Ruohua Zheng, "Stochastic fatigue crack growth in steel structures subject to random loading," Structural Safety, vol. 20, 303-323, (1998)

X. P. A. Preumont, "Spectral methods for multiaxial random fatigue analysis of metallic," International Journal of Fatigue, vol. 22, 541-550, (2000).

Y. Liu and S. Mahadevan, "Stochastic fatigue damage modeling under variable amplitude loading," International Journal of Fatigue vol. 29, pp. 1149-1161, 2006.

a. D. K. E. Macha and T. Łagoda and A. Niesłony, "Fatigue life under variable amplitude loading according to the cycle-counting and spectral methods," Materials Science, vol. 42, pp. 121-128, 2006.

R.tovo and D.benasciutti, "Frequency-based fatigue analysis of non-stationary switching random loads," Fatigue Fracture Engineering Material Structructure, vol. 30, pp. 10161029, 2007.

Mindlin R. D. "Influence of rotary inertia and shear in flexural motions of isotropic elastic plates" Journal of applied Mechanics, vol. 18, 31-38, (1951)

Moutaz M.Hegaze \& A. El-Zafrany " large deflection and stability analysis of composite layered plate using a new high-order element" Proceedings of the $11^{\text {th }}$ ASAT Conference, Cairo, Egypt, (2003)

El-Zafrany A. and Cookson R. A. "Derivation of Lagrangian and Hermitian shape functions for triangular elements" International Journal of Numerical Engineering, Vol. 23, 275-285, (1986a)

Daniel I. M. and Ishai O. "Engineering mechanics of composite materials" Oxford university press, Oxford, UK, (1994). 
Phan N. D. and Reddy J. N. "Analysis of laminated composite plates using a higher-order shear deformation theory" International Journal for Numerical Methods in Engineering, Vol. 21, 2201-2219, (1985).

I. Rychlik, "A new definition of the rainflow cycle counting method," international journal of fatigue, vol. 9, 119-121, (1987).

M. A. Miner, "Cumulative damage in fatigue," Journal of applied Mechanics, vol. 12, 159164, (1945).

Fuchs H. O. and Stephens S. I. "Metal fatigue in engineering" John Wiley \& Sons, Inc., USA, (1980). 